\title{
Correction to: Hepatitis B Surface Antigen Loss with Tenofovir Disoproxil Fumarate Plus Peginterferon Alfa-2a:Week 120 Analysis
}

\author{
Sang Hoon Ahn ${ }^{1} \cdot$ Patrick Marcellin ${ }^{2} \cdot$ Xiaoli Ma ${ }^{3}$. Florin A. Caruntu ${ }^{4} \cdot$ Won Young Tak ${ }^{5}$ Magdy Elkhashab ${ }^{6}$. \\ Wan-Long Chuang ${ }^{7} \cdot$ Fehmi Tabak $^{8} \cdot$ Rajiv Mehta $^{9} \cdot$ Jörg Petersen $^{10} \cdot$ William Guyer $^{11} \cdot$ Belinda Jump ${ }^{11} \cdot$ Alain Chan $^{11}$. \\ Mani Subramanian ${ }^{11}$. Gerald Crans ${ }^{11}$. Scott Fung ${ }^{12}$. Maria Buti ${ }^{13} \cdot$ Giovanni B. Gaeta $^{14}$ - Aric J. Hui ${ }^{15,16}$. \\ George Papatheodoridis ${ }^{17} \cdot$ Robert Flisiak $^{18} \cdot$ Henry L. Y. Chan ${ }^{19}$
}

Published online: 15 October 2018

(c) The Author(s) 2018

\section{Correction to: Digestive Diseases and Sciences https://doi.org/10.1007/s10620-018-5251-9}

The original version of this article unfortunately contained affiliation and textual errors. This has been corrected with this erratum.

Affiliation of eleventh author was incorrectly assigned. Dr. William Guyer is affiliated with Gilead Sciences Inc, Foster City, CA, USA.

In Results section, Safety Profile subsection, the sentence, 'Rates of serious adverse events were $11 \%, 10 \%, 7 \%$, and

The original article can be found online at https://doi.org/10.1007/ s10620-018-5251-9.

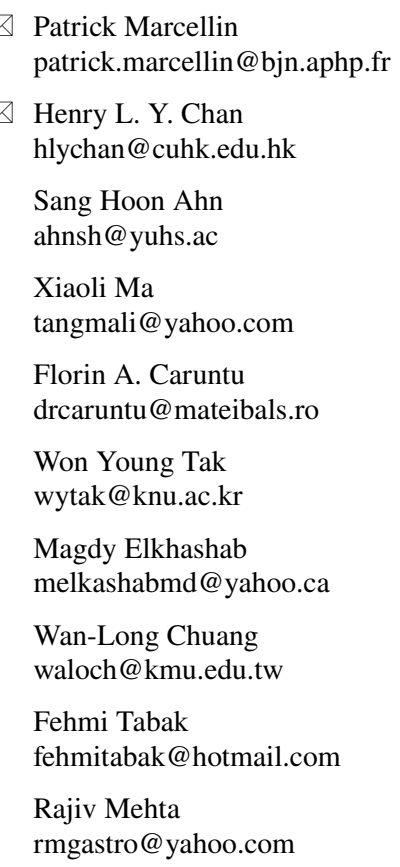

$10 \%$ in groups $\mathrm{A}, \mathrm{B}, \mathrm{C}$, and $\mathrm{D}$, respectively, and $6 \%, 3 \%$, and $5 \%$ in groups $\mathrm{A}, \mathrm{B}$, and $\mathrm{D}$, respectively, among those patients who were treated with TDF' should read as below:

Rates of serious adverse events were $11 \%, 10 \%, 7 \%$, and $10 \%$ in groups $\mathrm{A}, \mathrm{B}, \mathrm{C}$, and $\mathrm{D}$, respectively, and $6 \%, 3 \%$, and $5 \%$ in groups $\mathrm{A}, \mathrm{B}$, and $\mathrm{D}$, respectively, among those patients who were retreated with TDF.

In Table 2, header 'Mean HBV DNA change from baseline, $\log _{10} \mathrm{IU} / \mathrm{mL}$ (SD)' row 3 is missing ' $\mathrm{B}$ ' in genotype. Revised version of the Table 2 is given below.
Jörg Petersen

petersen@ifi-medizin.de

William Guyer

william.guyer@gilead.com

Belinda Jump

belinda.jump@gilead.com

Alain Chan

alain.chan@gilead.com

Mani Subramanian

mani.subramanian@gilead.com

Gerald Crans

gerald.crans@gilead.com

Scott Fung

scott.fung@uhn.on.ca

Maria Buti

mbuti@vhebron.net

Giovanni B. Gaeta

giovannibattista@unicampania.it

Aric J. Hui

arichui@yahoo.com 
Table 2 Efficacy results at week 120 by baseline HBeAg status and genotype

\begin{tabular}{|c|c|c|c|c|}
\hline Response & $\begin{array}{l}\text { Group A }(n=186) \\
\text { TDF+ PEG-IFN } \\
\text { for } 48 \text { weeks }\end{array}$ & $\begin{array}{l}\text { Group B } \\
(n=184) \\
\text { TDF+ PEG-IFN } \\
\text { for } 16 \text { weeks, } \\
\text { TDF for } \\
32 \text { weeks }\end{array}$ & $\begin{array}{l}\text { Group C }(n=185) \\
\text { TDF for } 120 \text { weeks }\end{array}$ & $\begin{array}{l}\text { Group D }(n=185) \\
\text { PEG-IFN } \\
\text { for } 48 \text { weeks }\end{array}$ \\
\hline \multicolumn{5}{|c|}{ HBsAg loss, Kaplan-Meier estimate (\%) } \\
\hline Overall & 10.36 & 3.49 & 0 & 3.51 \\
\hline $\mathrm{HBeAg}$ positive & 9.73 & 5.15 & 0 & 5.23 \\
\hline HBeAg negative & 10.99 & 1.32 & 0 & 1.28 \\
\hline \multicolumn{5}{|c|}{ HBsAg seroconversion, Kaplan-Meier estimate (\%) } \\
\hline Overall & 10.08 & 0.56 & 0 & 2.87 \\
\hline HBeAg positive & 10.41 & 0.97 & 0 & 4.10 \\
\hline HBeAg negative & 9.65 & 0 & 0 & 1.28 \\
\hline \multicolumn{5}{|c|}{ Mean HBsAg change from baseline, $\log _{10} \mathrm{IU} / \mathrm{mL}$ (SD) } \\
\hline Overall & $-2.4(2.4)$ & $-0.8(1.5)$ & $-0.4(0.7)$ & $-1.1(1.6)$ \\
\hline Genotype A & $-4.2(2.3)$ & - & $-0.7(1.2)$ & $0.0(0.6)$ \\
\hline Genotype B & $-2.2(2.1)$ & $-1.0(1.0)$ & $-0.7(0.6)$ & $-1.2(1.2)$ \\
\hline Genotype C & $-1.5(2.2)$ & $-0.4(1.5)$ & $-0.4(0.7)$ & $-1.1(1.7)$ \\
\hline Genotype D & $-2.0(2.3)$ & $-1.6(2.6)$ & $-0.2(0.4)$ & $-1.5(2.9)$ \\
\hline $\mathrm{HBeAg}$ positive & $-2.2(2.6)$ & $-1.2(1.9)$ & $-0.7(0.7)$ & $-1.3(2.1)$ \\
\hline HBeAg negative & $-2.5(2.1)$ & $-0.4(0.9)$ & $-0.1(0.4)$ & $-1.0(1.1)$ \\
\hline \multicolumn{5}{|c|}{ Mean HBV DNA change from baseline, $\log _{10} \mathrm{IU} / \mathrm{mL}$ (SD) } \\
\hline Overall & $-4.2(2.1)$ & $-4.3(2.0)$ & $-5.7(1.6)$ & $-3.5(2.0)$ \\
\hline Genotype A & $-4.0(1.9)$ & - & $-5.1(2.1)$ & $-1.9(2.0)$ \\
\hline Genotype B & $-4.7(2.0)$ & $-4.2(2.5)$ & $-5.8(1.4)$ & $-3.3(1.8)$ \\
\hline Genotype C & $-4.2(2.9)$ & $-4.4(1.5)$ & $-5.9(1.6)$ & $-3.4(2.3)$ \\
\hline Genotype D & $-4.0(1.8)$ & $-4.1(3.0)$ & $-5.3(1.7)$ & $-5.1(1.9)$ \\
\hline HBeAg positive & $-5.5(1.6)$ & $-5.3(1.5)$ & $-6.6(1.2)$ & $-4.9(1.6)$ \\
\hline HBeAg negative & $-2.9(1.7)$ & $-3.4(1.9)$ & $-4.5(1.3)$ & $-2.5(1.8)$ \\
\hline
\end{tabular}

George Papatheodoridis gepapath@med.uoa.gr

Robert Flisiak

robert.flisiak@umwb.edu.pl

1 Department of Internal Medicine, Yonsei University College of Medicine, Brain Korea 21 Plus Project for Medical Science, Seoul, Republic of Korea

2 Hôpital Beaujon, University Paris-Diderot, Clichy, France

3 Drexel University College of Medicine, Philadelphia, PA, USA

4 National Institute for Infectious Diseases "Matei Bals", Bucharest, Romania

5 Kyungpook National University Hospital, Daegu, South Korea

6 Toronto Liver Centre, Toronto, Canada

7 Kaohsiung Medical University Hospital, Kaohsiung Medical University, Kaohsiung, Taiwan

8 Cerrahpasa Medical Faculty, University of Istanbul, Istanbul, Turkey

9 Liver Clinic, Surat, India
10 IFI Institute for Interdisciplinary Medicine, Asklepios Klinik St. George, University of Hamburg, Hamburg, Germany

11 Gilead Sciences Inc, Foster City, CA, USA

12 Toronto General Hospital, Toronto, Canada

13 Hepatology Unit, Hospital Universitari Vall d'Hebron and CIBEREHD del Instituto Carlos III, Barcelona, Spain

14 Infectious Diseases and Viral Hepatitis Unit, University of Campania "Luigi Vanvitelli”, Naples, Italy

15 The Chinese University of Hong Kong, Hong Kong, China

16 Alice Ho Miu Ling Nethersole Hospital, Hong Kong, China

17 Medical School of National and Kapodistrian University of Athens, General Hospital of Athens "Laiko", Athens, Greece

18 Department of Infectious Diseases and Hepatology, Medical University of Bialystok, Białystok, Poland

19 Department of Medicine and Therapeutics and Institute of Digestive Disease, The Chinese University of Hong Kong, Hong Kong, China 